\title{
Routing Protocol Research for Wireless Quantum Networks based on Resource Reservation
}

\author{
Xinliang Wang ${ }^{\mathrm{a}, \mathrm{b},{ }^{*}}$, Qinggai Huang ${ }^{\mathrm{a}}$, Zhihuai Liu ${ }^{\mathrm{b}}, \mathrm{Na} \mathrm{Liu}{ }^{\mathrm{c}}$, and Wei Fang ${ }^{\mathrm{c}}$ \\ ${ }^{a}$ School of Physics and Electronic Information Engineering, Henan Polytechnic University, Jiaozuo, 454000, China \\ ${ }^{b}$ Hami Yuxin Energy Industry Research Institute Co., Ltd., Hami, 839000, China \\ ${ }^{c}$ Hami Vocational and Technical College, Hami, 839000, China
}

\begin{abstract}
In wireless quantum communication networks, existing AODV routing protocols can guarantee the stability of quantum entangled channels. However, the protocol takes a long time to establish the quantum channel on average, and it also consumes many entanglement resources. In order to solve the above problems, a routing protocol for wireless quantum networks based on resource reservation is proposed in this paper. Before the source node sends the quantum state information to the destination node, the proposed protocol estimates the quantum state information to be transmitted and writes the resource estimation value into the routing request message. A broadcast routing request message can not only satisfy the requirement of quantum data transmission, but also ensure that the number of hops is as small as possible. Simulation results show that the proposed protocol can effectively shorten the time of establishing quantum channels, reduce the consumption of entanglement resources, and improve the communication efficiency of wireless quantum communication networks. It has good practical value.
\end{abstract}

Keywords: quantum entanglement; routing metric; quantum communication network; resource reservation

(Submitted on October 25, 2018; Revised on November 27, 2018; Accepted on December 26, 2018)

(C) 2019 Totem Publisher, Inc. All rights reserved.

\section{Introduction}

Quantum communication is a subject that has been developed in recent years. It is impossible to clone [1] the unknown quantum state accurately, so there is high security for the transmitted information. In 1984, Yurke and Denker [2] proposed the concept of quantum communication networks. In the same year, Bennett and Brassard [3] verified the transfer-ability of the quantum states of the BB84 protocol by using the in-distinguish-ability principle of non-orthogonal quantum states. In 1997, Bouwmeester and Pan et al. [4] studied teleportation based on photons for the first time. The following year, Braunstein et al. [5] put forward a concrete scheme to realize the teleportation of quantum states with continuous variables. In 2009, Zhu et al. [6] used the balanced homodyne detection method to obtain the stable key generation rate of the continuous variable quantum key distribution protocol and used it in the design of quantum communication systems. In 2010, 16km quantum teleportation in free space was successfully achieved by Pan [7] of China University of Science and Technology and Tsinghua University. In 2012, Huang [8] made use of a two-particle entangled state to realize the teleportation of the three-particle entangled state, which was extended to $\mathrm{N}$ particle and saved quantum channel entanglement resources.

In recent years, with the research of quantum teleportation becoming increasingly mature, the design of routing protocols in quantum communication networks is becoming more and more perfect. In 2012, Yu et al. [9] proposed an ondemand routing protocol for wireless self-organized quantum communication networks, which can reduce the transmission resource of wireless channels. In the same year, Yang et al. [10] proposed the data link layer quantum selective retransmission protocol, which not only reduces the propagation delay in the communication process, but also improves the communication efficiency. In 2015, Ma et al. [11] proposed a quantum error-correcting code routing protocol for small 
quantum communication networks to achieve one-hop, two-hop, and secure quantum teleportation from source quantum nodes to destination nodes. In 2016, Wang [12] proposed a layered quantum packet transmission scheme, which can effectively improve the throughput of source nodes and the utilization of links in the network. In the same year, Wang [13] proposed a quantum teleportation routing protocol suitable for quantum wireless multi-hop networks, which can reduce transmission overhead, reduce transmission delay, and improve network lifetime. In 2017, Yuan et al. [14] proposed a relaybased routing protocol for quantum communication networks. The simulation results show that this protocol has strong operability. In 2017, Fan [15] constructed a quantum wireless communication network model, combined with Grover quantum search algorithm, to avoid the loss of quantum channels due to entangled quantum pairs and to reduce the computational complexity of quantum communication networks. Wang [16] proposed an adaptive quantum RIP algorithm on the basic principle of quantum communication, which realized the efficient, secure, and fast updating of routing information in autonomous systems and reduced the consumption of entangled resources as much as possible.

In this paper, the routing protocols and routing strategies of quantum communication networks are studied in detail. They can reduce the consumption of entanglement resources and the transmission delay of quantum state information in quantum communication networks to a certain extent. Based on the existing protocols, a resource reservation based routing protocol is proposed for wireless self-organized quantum communication networks. This protocol can not only save entanglement resources between nodes, but also shorten the transmission delay. It can improve the communication efficiency of wireless quantum communication networks and has good practical value.

\section{Routing Protocol for Wireless Quantum Networks based on Resource Reservation}

The establishment of quantum channels depends on the entangled particle pairs between nodes. In literature [9], the number of entangled particle pairs between nodes is taken as the routing metric. By comparing the routing measures in the routing request message with the routing metrics in the routing table, the intermediate node selects the minimum routing measures in each path.

However, the proposed wireless quantum network routing protocol based on resource reservation (protocol 1) is different from the AODV routing protocol (protocol 2) proposed in reference [9]. Before broadcasting the routing request, the source node estimates the quantum state information that the user needs to transmit and writes the resource reserve value into the routing request message as the routing measure of the routing request message. The routing resource reservation value is recorded as $N$. Assuming that the path from the source node to the destination node in a wireless quantum network is $n$, the routing measure between adjacent nodes is recorded as $N_{i j}(i=1,2,3, \cdots, q, j=1,2,3, \cdots, q)$, where $i$ represents the $i$ path from the source node to the destination node and $j$ represents the $j$ node on the $i$ path. Based on the resource reservation value saved $N$ in the routing request message and $N_{i j}$ routing selection from multiple paths, the routing process is as follows.

\subsection{Quantum Routing Broadcast and Response Protocol based on Resource Reservation}

In wireless quantum communication networks, the source node transmits quantum state information. If there is a valid route to the destination node in the source node routing table, quantum state information can be transmitted directly; if not, the source node broadcasts a routing request message RREQ. The data to be sent by the user is estimated, and the estimated value of the resource is $N$ written into the routing request message RREQ. The routing request message format is shown in Table 1, and the routing process is as follows:

Table 1. Resource reservation routing request message

\begin{tabular}{|c|}
\hline Routing metric $N$ \\
\hline Last hop node address \\
\hline Source node address \\
\hline Destination node address \\
\hline Source node sequence number \\
\hline
\end{tabular}

Step 1 After the source node broadcasts the route request message, the intermediate node determines whether the route request message has been received according to the source node sequence number of the route request message. If the route request message has been received, the route request message is discarded. If the route request message has not been received, the source node sequence number is stored in the node routing table.

Step 2 The intermediate Node1 compares the reserved resource value $N$ in the routing request message with the 
routing measure $N_{i j}$ in the routing table. If $N$ is greater than $N_{i j}$, the entangled resource between $j-1$ and $j$ nodes in the path $i$ is unable to transmit quantum state information, and the routing request message will stop broadcast at the node Node1. If $N$ is less than or equal to $N_{i j}$, the network resources between the $j-1$ and $j$ nodes in the path are capable of transmitting the quantum state information to be transmitted by the source node.

Step 3 Update the Node1 routing table. First, the intermediate node Node1 checks whether the route from the routing table to the source node is valid. If valid, and if the routing measure value $N_{i j}$ in the routing table is greater than or equal to the reserved resource value $N$ in the routing request message, the next hop node address in the routing table is the address of the previous hop node in the routing request message. If the routing measure $N_{i j}$ in the routing table is less than the reserved resource value $N$ in the routing request message, the routing table is not updated. If the routing table from the intermediate node to the source node is invalid, the effective route is added to the source node according to the address of the source node in the routing broadcast request message.

Step 4 Update the address of the last hop node in the routing request message to Node 1, add 1 hop, and continue broadcasting the routing request message.

Step 5 After receiving the routing request message sent by the neighboring nodes, the destination node selects a transmission path with the smallest hop number among the multiple alternative paths and then sends the routing reply message to the destination node. The format of the routing reply message is shown in Table 2.

Table 2. Routing reply message format

\begin{tabular}{|c|c|}
\hline Path node numbers & Hop number \\
\hline Destination node address \\
\hline Source node address \\
\hline Last hop node address \\
\hline
\end{tabular}

Step 6 In the route reply message sent by the destination node, the number of path nodes is the number of hops of the selected path, and the address of the previous hop node is the address of the previous hop node in the routing request message. Then, update the address of the previous hop node to the next hop node address in the routing table, and add 1 hop. After the source node receives the routing response message, the route selected by the destination node is added to its routing table to complete the routing request and response process and determine the quantum state information transmission path. Finally, quantum information transmission is accomplished by establishing the quantum channel.

\subsection{Routing Maintenance Algorithm based on Entangled Resource Compensation}

The establishment of the quantum channel requires the consumption of quantum entanglement pairs between nodes. Each time the source node transmits quantum state information, the entangled particle pairs between nodes will be reduced, and eventually the quantum channel will not be established for other users. The quantum state cannot be transmitted. In order to solve the above problem, this paper proposes a route maintenance algorithm based on entangled resource compensation. The concrete steps are as follows:

Step 1 Node2 periodically sends the HELLO message to the adjacent Node3, and the resource maintenance value in the message is $S$. When the adjacent Node3 receives the message, it indicates that Node2 and the Node3 are reachable, and step 2 is executed. If the adjacent Node 3 does not receive the message, step 4 is executed.

Step 2 Node3 determines whether entanglement resources between nodes need to be compensated. Node3 compares the size of the resource maintenance value $S$ in the HELLO packet with the routing metric $N_{i j}$ in the routing table. If $S$ is greater than or equal to the routing metric $N_{i j}$, it means that there are fewer pairs of entangled particles between two nodes, which will affect the subsequent transmission of quantum information, so the entangled resources between nodes need to be compensated, and step 3 is executed. On the other hand, if $S$ is less than the routing measure $N_{i j}$, there is no need to compensate the entangled resources between nodes.

Step 3 A pair of $n$ entangled particles is prepared by Node 3 and represented as $A_{i} B_{i}$, where $1 \leq i \leq n$; the entangled 
state of each pair of entangled particles $A_{i} B_{i}$ is expressed as $\chi_{A_{i} B_{i}}=\alpha|01\rangle_{A_{i} B_{i}}+\beta|10\rangle_{A_{i} B_{i}}$, where $\alpha^{2}+\beta^{2}=1$. Leave the particle $A_{i}$ to the node Node3, and send the particle $B_{i}$ to Node2.

\section{Quantum Data Transmission Scheme based on Resource Reservation Routing}

After the source node completes the routing discovery process, the quantum entanglement channel is established and the quantum state information is transmitted. The topology of quantum wireless networks is shown in Figure 1.

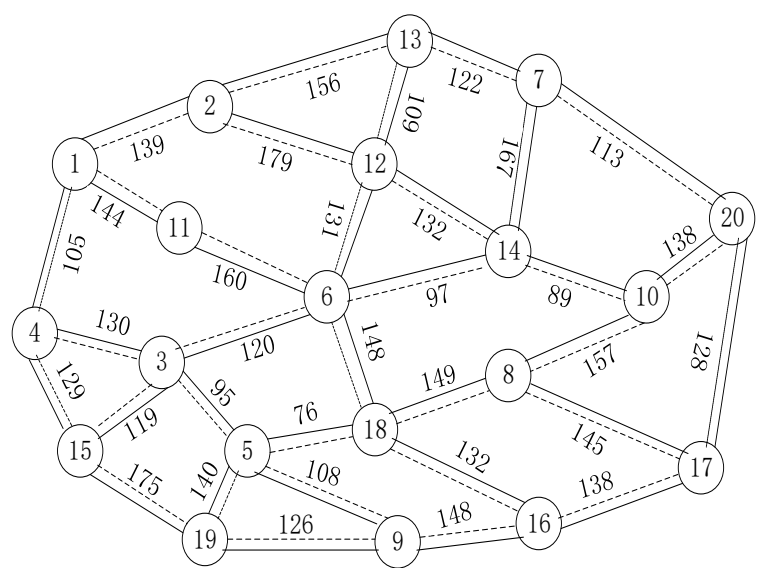

Figure 1. Topology of quantum wireless networks

At some point, node (1) transmits quantum state information to node (20). According to the AODV routing protocol (protocol2) proposed in reference [9], the path with larger routing measures is chosen among the optional paths. Figure 2 shows that the path chosen is $(1)-11-6-(18-8-10-20$. The proposed resource reservation-based quantum routing protocol (protocol1) estimates the quantum state information to be transmitted and assumes that the resource reservation value $N$ is 100qbit. From Figure 2, we can see that the minimum hop path is $1-2-13-7-20$. After the routing is completed, a quantum channel is established between the source node (1) and the destination node 20. The specific process of establishing the quantum channel is as follows.

\subsection{Building Quantum Channel based on Path Selection for Resource Reservation Routing}

For the network topology shown in Figure 1, the path of resource reservation routing selection is $(1)-(2)-(13)-(7)-(20)$. In this paper, the method of two-end approximation [9] is used to establish the quantum channel between the source node and the destination node, which can reduce the number of measurements of quantum entangled states. Based on the path of resource reservation routing, the entanglement state between nodes is shown in Figure 2. The specific steps of quantum channel establishment are as follows:

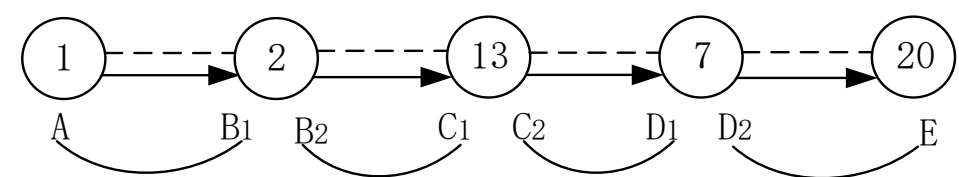

Figure 2. Quantum channel constructed based on two-terminal approximation method

Step 1 Based on the path chosen by resource reservation routing, the entangled states between nodes are as follows:

$$
\begin{aligned}
& \left|\Phi^{+}\right\rangle_{A B_{1}}=\frac{1}{\sqrt{2}}\left(|01\rangle_{A B_{1}}+|10\rangle_{A B_{1}}\right), \quad\left|\Phi^{+}\right\rangle_{B_{2} C_{1}}=\frac{1}{\sqrt{2}}\left(|01\rangle_{B_{2} C_{1}}+|10\rangle_{B_{2} C_{1}}\right) \\
& \left|\Phi^{+}\right\rangle_{C_{2} D_{1}}=\frac{1}{\sqrt{2}}\left(|01\rangle_{C_{2} D_{1}}+|10\rangle_{C_{2} D_{1}}\right), \quad\left|\Phi^{+}\right\rangle_{D_{2} E}=\frac{1}{\sqrt{2}}\left(|01\rangle_{D_{2} E}+|10\rangle_{D_{2} E}\right)
\end{aligned}
$$

Step 2 The entangled states of particles $A, B_{1}, B_{2}, C_{1}$ in nodes (1), 2), (13) are shown in Equation (1): 


$$
\begin{aligned}
|\phi\rangle_{A B_{1} B_{2} C 1} & =\left|\Phi^{+}\right\rangle_{A B_{1}} \otimes\left|\Phi^{+}\right\rangle_{B_{2} C_{1}} \\
& =\frac{1}{2}\left(|0101\rangle_{A B_{1} B_{2} C_{1}}+|0110\rangle_{A B_{1} B_{2} C_{1}}+|1001\rangle_{A B_{1} B_{2} C_{1}}+|1010\rangle_{A B_{1} B_{2} C_{1}}\right)
\end{aligned}
$$

The particles $B_{1}$ and $B_{2}$ of node (2) pass through the CONT gate, that is:

$$
|00\rangle \rightarrow|00\rangle,|01\rangle \rightarrow|01\rangle,|10\rangle \rightarrow|11\rangle,|11\rangle \rightarrow|10\rangle
$$

Then, they are sent to the $\mathrm{H}$ gate, that is:

$$
|0\rangle \rightarrow \frac{1}{\sqrt{2}}(|0\rangle+|1\rangle),|1\rangle \rightarrow \frac{1}{\sqrt{2}}(|0\rangle-|1\rangle)
$$

After transformation, the state of the four particles is shown in Equation (2):

$$
\begin{aligned}
|\phi\rangle_{A B_{1} B_{2} C 1} & =\frac{1}{2 \sqrt{2}}\left[|00\rangle_{B_{1} B_{2}}\left(|01\rangle_{A C_{1}}-|10\rangle_{A C_{1}}\right)+|01\rangle_{B_{1} B_{2}}\left(|00\rangle_{A C_{1}}+|11\rangle_{A C_{1}}\right)\right. \\
& \left.+|10\rangle_{B_{1} B_{2}}\left(|00\rangle_{A C_{1}}-|11\rangle_{A C_{1}}\right)+|11\rangle_{B_{1} B_{2}}\left(|01\rangle_{A C_{1}}+|10\rangle_{A C_{1}}\right)\right]
\end{aligned}
$$

Node (2) measures the transformed particles $B_{1}, B_{2}$ based on BELL and sends the measurement results to the node (13) classical wireless channel. After the corresponding gate operation, the particles $C_{1}, D_{2}, D_{2}, E$ in the entangled state $|\phi\rangle_{C_{2} D_{1} D_{2} E}$ formed by the same node $\left(7\right.$ are obtained. The transformed particles $D_{1}, D_{2}$ are also measured by Bell, the measurement result is sent to node 13 through the classical channel, and the third step is executed.

Step 3 After node (13) receives the measurements of nodes (2) and (7), if particles $A, C_{1}$ are in an entangled state $\left|\Phi^{+}\right\rangle_{A C_{1}}=\frac{1}{\sqrt{2}}\left(|01\rangle_{A C_{1}}+|10\rangle_{A C_{1}}\right)$ and particles $C_{2}, E$ are in an entangled state $\left|\Phi^{-}\right\rangle_{C_{2} E}=\frac{1}{\sqrt{2}}\left(|01\rangle_{C_{2} E}-|10\rangle_{C_{2} E}\right)$, then the entangled state of the four particles is shown in Equation (3):

$$
|\phi\rangle_{A C_{1} C_{2} E}=\frac{1}{2}\left(|0101\rangle_{A C_{1} C_{2} E}-|0110\rangle_{A C_{1} C_{2} E}+|1001\rangle_{A C_{1} C_{2} E}-|1010\rangle_{A C_{1} C_{2} E}\right)
$$

As with the transformation in step 2, the particles $C_{1}, C_{2}$ are passed through the CONT and $\mathrm{H}$ gates, and the entangled state is shown in Equation (4):

$$
\begin{aligned}
|\phi\rangle_{A C_{1} C_{2} E} & =\frac{1}{2}\left[|00\rangle_{C_{1} C_{2}}\left(|01\rangle_{A E}-|10\rangle_{A E}\right)-|01\rangle_{C_{1} C_{2}}\left(|00\rangle_{A E}+|11\rangle_{A E}\right)\right. \\
& \left.+|10\rangle_{C_{1} C_{2}}\left(|00\rangle_{A E}-|11\rangle_{A E}\right)-|11\rangle_{C_{1} C_{2}}\left(|01\rangle_{A E}+|10\rangle_{A E}\right)\right]
\end{aligned}
$$

Node (13) measures the particles $C_{1}, C_{2}$ based on Bell and sends the measurement results to source node (1), the classical wireless channel. After that, the establishment of the quantum channel is completed.

\subsection{Quantum Data Transmission Scheme based on Resource Reservation Routing and Routing Path}

After the establishment of the quantum channel, node (1) begins to transmit quantum state information, assuming that the entangled state constructed by particles $A, E$ is $\left|\varphi^{+}\right\rangle_{A E}=\frac{1}{\sqrt{2}}\left(|00\rangle_{A E}+|11\rangle_{A E}\right)$. The source node encodes 0 into $|0\rangle$ state and 1 into $|1\rangle$ state. The encoded quantum state information is $|\varphi\rangle=\alpha|0\rangle+\beta|1\rangle\left(\alpha^{2}+\beta^{2}=1\right)$. The source node measures 
$|\varphi\rangle$ with particle $|\Phi\rangle_{A E}$ in the Bell state, which is equivalent to $|\varphi\rangle$ having a direct product with particle $|\Phi\rangle_{A E}$. It can be expressed as Equation (5):

$$
\begin{aligned}
|\varphi\rangle\left|\varphi^{+}\right\rangle_{A E} & =(\alpha|0\rangle+\beta|1\rangle) \otimes \frac{1}{\sqrt{2}}\left(|00\rangle_{A E}+|11\rangle_{A E}\right) \\
& =\frac{\alpha}{\sqrt{2}}(|000\rangle+|011\rangle)+\frac{\beta}{\sqrt{2}}(|100\rangle+|111\rangle)
\end{aligned}
$$

The source node will feed particle $|\varphi\rangle$ and particle $A$ into the controlled gate, and Equation (5) can be expressed as the following Equation (6):

$$
\begin{aligned}
|\varphi\rangle\left|\varphi^{+}\right\rangle_{A E} & =\frac{\alpha}{\sqrt{2}}(|000\rangle+|011\rangle)+\frac{\beta}{\sqrt{2}}(|110\rangle+|101\rangle) \\
& =\frac{\alpha}{\sqrt{2}}|0\rangle(|00\rangle+|11\rangle)+\frac{\beta}{\sqrt{2}}|1\rangle(|10\rangle+|01\rangle)
\end{aligned}
$$

Then, the first quantum state information is fed into the $\mathrm{H}$ gate, and the final state is shown in Equation (7):

$$
\begin{aligned}
|\varphi\rangle\left|\varphi^{+}\right\rangle_{A E} & =\frac{\alpha}{2}(|0\rangle+|1\rangle)(|00\rangle+|11\rangle)+\frac{\beta}{2}(|0\rangle-|1\rangle)(|10\rangle+|01\rangle) \\
& =\frac{1}{2}|00\rangle(\alpha|0\rangle+\beta|1\rangle)+|10\rangle(\alpha|0\rangle-\beta|1\rangle)-|11\rangle(\alpha|1\rangle-\beta|0\rangle)+|01\rangle(\alpha|1\rangle+\beta|0\rangle)
\end{aligned}
$$

Node (1) was used to measure the Bell basis of particles $A, E$. The measured results were $|00\rangle,|01\rangle,|10\rangle,|11\rangle$. Node (1) transmits the measurement results to node (20) by the classical wireless channel. Then, node (20) transmits the results according to node (1) to make the corresponding unitary transformation according to Table 3 to restore the quantum state information.

Table 3. Router restoration quantum state information reference table

\begin{tabular}{c|c|c}
\hline Node measurement results & Information issued & The corresponding operation of the destination node \\
\hline$|00\rangle$ & 00 & Through the $X$ gate \\
\hline$|01\rangle$ & 01 & Do nothing \\
\hline$|10\rangle$ & 10 & Through the $X$ door, then through the $Z$ door \\
\hline$|11\rangle$ & 11 & Through the $Z$ gate \\
\hline
\end{tabular}

\section{Simulation Analysis}

A resource reservation-based routing protocol (protocol1) for wireless quantum communication is proposed in this paper. Before the source node broadcasts the routing request message, the quantum state information to be transmitted is estimated, and the network reserves entangled resources for the transmission of the data by broadcasting the routing request message to ensure that the quantum channel established when the source node transmits quantum state information to the destination node is not interrupted.

In this paper, the NS2 simulation environment is used to simulate the performance of routing protocol protocoll. Because NS2 has its own network node mobile generation scene file and service generation scene file, in the wireless network simulation environment, the network node is distributed in the range of $1000 \times 1000 \mathrm{~m}^{2}$. Within the range of effective wireless transmission distance, there are random integers between 50-200 pairs of entangled particles between nodes, and the resource reservation value in the routing request message is set to $N=100$ qbit .

By changing the number of nodes in the network and analyzing the trace files generated by the Tool Command 
Language script, we can see that as the number of nodes in the network increases, compared with the AODV routing protocol (protocol2) proposed in [9], the proposed wireless quantum communication network routing protocol (protocol1) based on resource reservation can effectively reduce the number of selected routing nodes when the source node transmits quantum state information to the destination node, as shown in Figure 3:

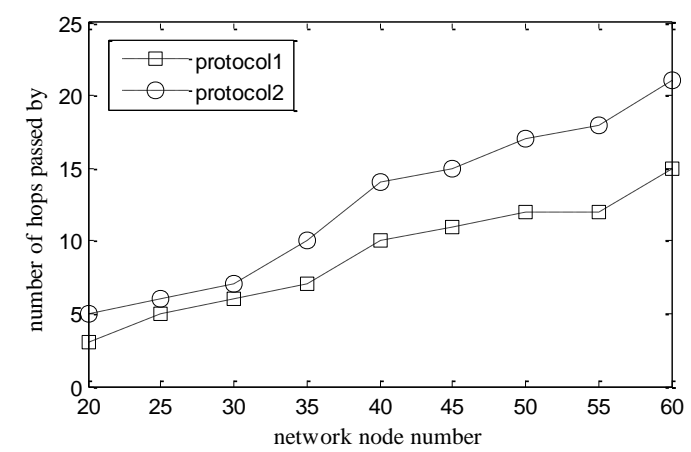

Figure 3. The number of hops established by different network sizes

Figure 3 shows that the number of hops from the source node to the destination node increases as the network size increases. With the same number of nodes in the network, the proposed routing scheme based on resource reservation (protocol1) is compared with the on-demand routing protocol (protocol2) proposed in reference [9]. When the source node transmits the quantum state information to the destination node, the number of intermediate nodes passing through is relatively small. It can be concluded that protocoll can effectively reduce the intermediate link of data transmission while ensuring the stability of the quantum channel.

Quantum information transmission must rely on quantum channels. In the process of establishing quantum channels, the entangled particle states need to be measured based on Bell, and the measurement results are transmitted to the corresponding nodes via the classical wireless channel. Assuming that the source node determines the transmission path, the number of nodes in the path is $m$. In the process of establishing quantum channels by using the two-end approximation [9] method, when $m$ is odd, the total number of measurements in the entangled states during establishment of entangled channels is shown in Equation (8):

$$
\kappa_{1}=\left\lfloor\frac{2 m-1}{2}\right\rfloor-1
$$

When $m$ is even, the total number of measurements in the entangled states during establishment of entangled channels is shown in Equation (9):

$$
\kappa_{2}=\left\lfloor\frac{m-1}{2}\right\rfloor+\frac{m-2}{2}
$$

It is assumed that in the process of establishing the quantum channel, the time of Bell basis measurement of particles is $t_{s}$, the average time of transmission of measurement results in the classical wireless channel is $t_{r}$, and the average time for the source node to recover from the measured results by unitary transformation is $T_{R}$. When $m$ is odd, the average time of establishing the quantum entangled channel is shown in Equation (10):

$$
T=\left(t_{s}+t_{r}+T_{R}\right) \cdot \kappa_{1}=\left(t_{s}+t_{r}+T_{R}\right) \cdot\left(\left\lfloor\frac{2 m-1}{2}\right\rfloor-1\right)
$$

When $m$ is even, the average time of establishing the quantum entanglement channel is shown in Equation (11):

$$
\left.T=\left(t_{s}+t_{r}+T_{R}\right) \cdot \kappa_{2}=\left(t_{s}+t_{r}+T_{R}\right) \cdot\left(\frac{m-1}{2}\right\rfloor+\frac{m-2}{2}\right)
$$


Assume that $t_{r}=10 \mathrm{~ns}, T_{R}=0.3 \mathrm{~ns}, t_{s}=0.2 \mathrm{~ns}$. Then, for the proposed quantum routing protocol (protocol1) based on resource reservation and the AODV routing protocol (protocol2) proposed in reference [9], the average time for establishing quantum entanglement channels is shown in Figure 4:

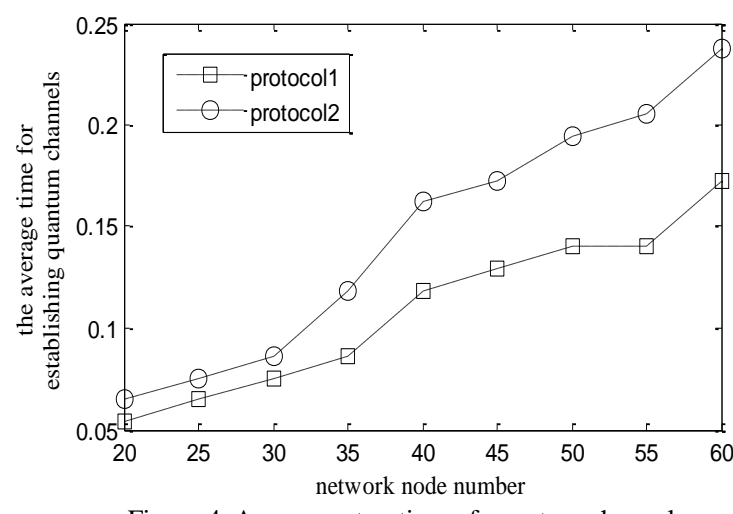

Figure 4. Average setup time of quantum channel

As shown in Figure 4, the average time for protocol1 and protocol2 to establish a quantum channel increases as the network size increases. With the same network size, the proposed routing protocol (protocol1) passes through a relatively small number of intermediate nodes, so the number of measurements of entangled states and the average time for establishing a quantum channel are lower.

In the process of constructing the quantum entanglement channel, entanglement particle pairs between nodes will be consumed. When the nodes $m$ in the path are odd, the relationship between entanglement resources consumed by the source node transmitting quantum state information once and the number of nodes in the path is shown in Equation (12):

$$
c=\left\lfloor\frac{m}{2}\right\rfloor+\frac{m-1}{2}
$$

When the nodes $m$ in the path are even, the relationship is shown in Equation (13):

$$
c=\left\lfloor\frac{m-1}{2}\right\rfloor+\frac{m}{2}
$$

Assuming that the source node needs to transmit 100 q-bits, compared with reference [9], the proposed routing protocol based on resource reservation consumes entangled resources as shown in Figure 5:

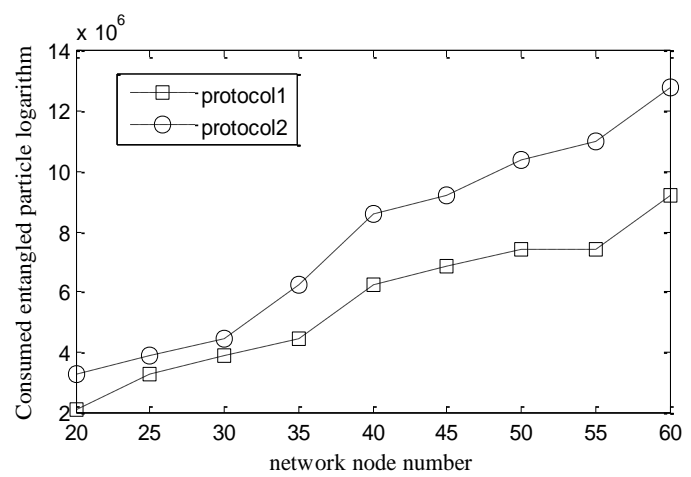

Figure 5. The relationship between the consumption of entangled particle pairs and the number of nodes in the network

As shown in Figure 5, as the number of network nodes increases, the network entanglement resources consumed by the transmission of quantum state information from the source node to the destination node increase gradually. When the network size is the same, the quantum routing protocol (protocol1) proposed in this paper can effectively reduce the 
consumption of entangled resources compared with the on-demand routing protocol (protocol2) proposed in reference [9].

In summary, compared with protocol2, the proposed resource reserved quantum routing protocol (protocol1) can effectively shorten the time of establishing the quantum channel, reduce the consumption of entanglement resources, and improve the communication efficiency of the wireless quantum communication network. It has good practical value.

\section{Conclusions}

Quantum communication networks are maintained by various quantum routing protocols, and the teleportation of quantum state information requires the establishment of quantum entanglement channels between nodes, which must consume entangled resources in the network. In this paper, resource reservation based on routing protocol for wireless quantum communication networks is proposed. Before the source node broadcasts the routing request message, the quantum state information transmitted to the destination node is used as the resource prediction. The resource revaluation is written into the routing request message, and the route discovery process is completed based on the resource revaluation. Simulation results show that the proposed routing protocol can reduce the number of hops between the source node and the destination node, shorten the time of establishing the quantum channel, and reduce the consumption of entangled resources in the network. It is of great practical value to improve the communication efficiency of wireless quantum communication networks.

\section{Acknowledgements}

This paper is supported by the Support Project of Science and Technology for Xinjiang Autonomous Region (No. 2018E02073), Doctor Fund of Henan Polytechnic University (No. B2012-073), Key Lab of Mine Informatization, Henan Polytechnic University (No. KY2015-08), Key Science and Technology Program of Henan Province (No. 172102210274), and 2018 Annual Funding Plan for Key Scientific Research Projects in Henan Universities (No. 18A470013).

\section{References}

1. W. K. Wootters and W. H. Zurek, “A Single Quantum Cannot be Cloned,” Nature, Vol. 299, No. 5886, pp. 802-803, 1982

2. B. Yurke and J. S. Denker, "Quantum Network Theory," Physical Review A, Vol. 29, No. 29, pp. 1419-1437, 1984

3. C. H. Bennett and G. Brassard, "Quantum Cryptography: Public Key Distribution and Coin Tossing," in Proceedings of the IEEE International Conference on Computers, Systems and Signal Processing, pp. 175-179, IEEE Press, New York, 1984

4. D. Bouwmeester, J. W. Pan, K. Mattle, M. Eibl, H. Weinfurter, and A Zeilinger, "Experimental Quantum Teleportation," Nature, Vol. 390, No. 6660, pp. 575-579, 1997

5. S. L. Braunstein and H. J. Kimble, "Teleportation of Continuous Quantum Variables," Physical Review Letters, Vol. 80, No. 4, pp. 869,1998

6. C. H. Zhu, C. X. Pei, D. X. Quan, N. Chen, and Y. H. Yi, “Adaptive Continuous Variable Quantum Key Distribution based on Channel Estimation,” Acta Physica Sinica, Vol. 58, No. 4, pp. 2184-2188, 2009

7. X. M. Jin, J. G. Ren, B. Yang, Z. H. Yi, X. F. Xu, et al., "Experimental Free-Space Quantum Teleportation," Nature Photonics, Vol. 4, No. 6, pp. 376-381, 2010

8. H. M. Huang, "Teleportation of N-Particle Entangled HGZ Sate via Two-Particle Entangled Quantum Channel," Chinese Journal of Quantum Electronics, Vol. 29, No. 6, 2012

9. X. T. Yu, J. Xu, and Z. C. Zhang, "Routing Protocol for Wireless Ad hoc Quantum Communication Network based on Quantum Teleportation," Acta Physica Sinica, Vol. 61, No. 22, 2012

10. X. L. Yang, X. Q. Zhou, H. Zhao, and P. P. Wang, "Data Link Layer of Selective Repeat Protocol based on Quantum Teleportation," Acta Physica Sinica, Vol. 61, No. 2, 2012

11. H. Y. MA, Z. W. Guo, X. K. Fan, and S. M. WANG, "The Routing Communication Protocol for Small Quantum Network based on Quantum Error Correction Code," Acta Electronica Sinica, Vol. 43, No. 1, 2015

12. L. F. Wang, "A Study for Packet Transmission Strategy of Quantum Communication," Xi'an University of Posts \& Telecommunications, 2016

13. K. Wang, "Research on Key Technologies of Quantum Wireless Multi-Hop Network," Southeast University, 2016

14. X. H. Yuan and C. W. Li, "Routing Protocol of Fiber Quantum Communication Network," Control Theory \& Application, Vol. 34, No. 11, 2017

15. X. C. Fan, K. Y. Liu, and X. J. Wen, "Communication Transmission and Routing Protocols of Quantum Mobile Internet," Chinese Journal of Quantum Electronics, Vol. 34, No. 5, 2017

16. X. Wang, Q. G. Haung, and W. Zhang, "An Adaptive RIP Routing Protocol based on Quantum Invisible Transmission," CN107070796A, 2017

Xinliang Wang received his Ph.D. in signal and information processing from Beijing University of Posts and Telecommunications in 2011. He is currently an associate professor in the School of Physics \& Electronic Information 
Engineering at Henan Polytechnic University. His current research interests include smart power grids, cloud computing, and big data.

Qinggai Huang received her Master's degree from Luoyang Institute of Science and Technology. She is currently a student in the School of Physics \& Electronic Information Engineering at Henan Polytechnic University. Her current research interests include quantum communication.

Zhihuai Liu works at Hami Yuxin Energy Industry Research Institute Co., Ltd. His research interests include cloud computing and big data.

Na Liu works at Hami Vocational and Technical College. Her research interests include cloud computing and big data.

Wei Fang works at Hami Vocational and Technical College. His research interests include smart power grids, cloud computing, and big data.

Xuebin Liu works at Hami Vocational and Technical College. His research interests include smart power grids, cloud computing, and big data.

Jun Wu works in the School of Physics and Electronic Information Engineering at Henan Polytechnic University. His current research interests include smart power grids, cloud computing, and big data. 